\title{
Percutaneous, Transtrapezial Fixation without Bone Graft Leads to Consolidation in Selected Cases of Delayed Union of the Scaphoid Waist
}

\author{
Matthias Vanhees, MD, PhD ${ }^{1,2}$ Roger R.P. van Riet, MD, $\mathrm{PhD}^{1,2}$ Annemieke van Haver, $\mathrm{PhD}^{3}$
}

Radek Kebrle, $M^{4}$ Geert Meermans, MD $^{5}$ Frederik Verstreken, MD ${ }^{1,2}$

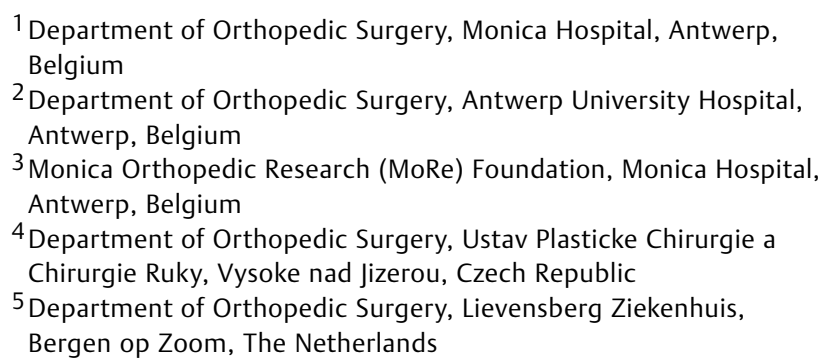

Address for correspondence Frederik Verstreken, MD, Department of Orthopedic Surgery, Monica Hospital, Stevenslei 20, 2100 Antwerp, Belgium (e-mail: frederik.verstreken@azmonica.be).

J Wrist Surg 2017;6:183-187.

\begin{abstract}
Keywords

- scaphoid fractures

- delayed union

- percutaneous fixation

- consolidation

Purpose We evaluated clinical and radiographic outcome of percutaneous transtrapezial fixation of the scaphoid delayed union or nonunion using a headless bone screw without bone grafting.

Methods Sixteen patients with delayed union or nonunion of the scaphoid were included in this retrospective study between 2006 and 2011. All patients had a delayed presentation of scaphoid fracture, and none of them was treated conservatively elsewhere. Patients with bone graft, sclerotic bone debridement, or displacement of the fragment at the nonunion site were excluded. A percutaneous transtrapezial fixation technique was used in all cases. Patients were reviewed until clinical and radiographic union was observed. At the final follow-up, DASH (Disabilities of the Arm, Shoulder and Hand) and PRWHE (Patient-Rated Wrist and Hand Evaluation) outcome scores were completed.

Results Radiographic union was obtained in 15 out of 16 patients (94\%) at an average follow-up of 36 months (range: 12-98 months). No complications from the percutaneous technique were noted. The average DASH score was 6 (range: $0-39$ ) and the average PRWHE score was 10 (range: $0-56$ ). No statistical significant difference in range of motion and grip strength was found between the operated side and the contralateral side.

Conclusion Percutaneous transtrapezial screw fixation for delayed or nonunion of selected scaphoid fractures without bone grafting is promising. At a mean of 4 months, $94 \%$ union was obtained with good functional results when there was no sclerosis, minimal osteolysis, and no displacement at the scaphoid nonunion site.

Type of Study Therapeutic study.

Level of Evidence IV.
\end{abstract}

received

June 27, 2016

accepted after revision

November 19, 2016

published online

December 28, 2016
Copyright $\odot 2017$ by Thieme Medical Publishers, Inc., 333 Seventh Avenue, New York, NY 10001, USA. Tel: +1(212) 584-4662.
DOI https://doi.org/

10.1055/s-0036-1597681. ISSN 2163-3916. 
Scaphoid fractures are the most common carpal fractures and carry a high risk of delayed union and pseudarthrosis. The incidence of scaphoid nonunion is between 5 and $25 \% .^{1-3}$ Untreated nonunion will lead to degenerative changes and scaphoid nonunion advanced collapse. ${ }^{4}$

Treatment options for delayed union of the scaphoid consist of cast immobilization ${ }^{5}$ or surgical treatment. Surgical options vary from percutaneous fixation to open reduction and internal fixation with or without bone grafting. Open reduction and internal fixation of the scaphoid has a higher complication rate, including scar problems, infection, nerve injury, and complex regional pain syndrome, ${ }^{6}$ compared with percutaneous fixation that is much safer and a minimally invasive technique. However, only limited data are available for the use of percutaneous fixation in cases of delayed union of the scaphoid, ${ }^{7,8}$ and it is often considered to be contraindicated. ${ }^{4}$

The definition of delayed presentation, delayed union, and nonunion are slightly variable in the literature. In this article, delayed presentation was defined as a lack of any sign of union 1 to 3 months postinjury. The cutoff between delayed union and nonunion was set at 6 months postinjury. ${ }^{9-12}$

The purpose of this study is to evaluate the use of transtrapezial percutaneous fixation with a headless bone screw without bone graft in a selected group of patients with delayed presentation, delayed union, or nonunion of a scaphoid fracture.

\section{Material and Methods}

A retrospective study was performed to analyze the outcome of the percutaneous fixation of selected cases of delayed presentation, delayed union, or nonunion of the scaphoid waist in the period between 2006 and 2011. Sixteen patients were included in this study: fifteen males and one female with an average age of 27 years (range: 17-61 years). The average time between trauma and surgery was 5 months (range: 2-27 months). A delayed presentation was present in six patients, a delayed union in eight, and a nonunion in two. The scaphoids nonunion was graded according to the revised classification of scaphoid nonunion. ${ }^{13}$ Two patients were categorized as grade 1,2 as grade 2 , and 12 as grade 3 nonunion. All cases were untreated late presentations of scaphoid fractures. In seven patients, computed tomography (CT) scans of the scaphoid were obtained to confirm the diagnosis and to decide on further treatment. Patients who needed bone graft, with sclerotic bone changes at the fracture site or cortical bone resorption with a displacement of more than $2 \mathrm{~mm}$, were excluded from the study based on the preoperative radiographic assessment. According to the Slade and Geissler scaphoid nonunion classification system, all class IV to VI nonunions were excluded from this study ${ }^{13}$ as well.

A percutaneous, transtrapezial approach was used in all cases to obtain central placement of the screw. The surgical technique was previously described already. ${ }^{14}$ The central axis of the scaphoid was determined with a guidewire under fluoroscopy and marked on the skin. Then, a guidewire was drilled through the trapezium, along the central axis of the scaphoid into the proximal cortex of the scaphoid. To determine the appropriate length of the screw, a second guidewire was drilled trough the trapezium into the distal cortex of the scaphoid. The difference in length between these two guidewires was set as the length of the screw needed. Finally, the guidewire was overdrilled with a 2-mm drill, and the headless Headless Bone Screw screw (KLS Martin, Tütlingen, Germany) was inserted.

Postoperatively, all patients were immobilized with a splint and were reviewed every 4 weeks until clinical and radiographic union was observed (-Figs. 1 and 2). Between June 2010 and May 2013, they were invited to visit the outpatient clinic for a clinical examination (including grip strength and range of motion [ROM]) and radiographic examination; DASH (Disabilities of the Arm, Shoulder and Hand) and PRWHE (Patient-Rated Wrist and Hand Evaluation) outcome scores were also obtained. All preoperative and final follow-up radiographs were taken to analyze degenerative changes on scaphotrapezial arthritis using the modified Eaton and Glickel classification. ${ }^{15}$

\section{Statistics}

Nonparametric statistical tests were applied to account for small sample size. Spearman's rho test was used to investigate if the time to surgery was related to the time to union. A p-value less than 0.05 was considered statistically significant.

We analyzed the grip strength and ROM (flexion, extension, pronation, supination, radial deviation, and ulnar deviation) using the paired samples Wilcoxon signed-rank test. The seven parameters were analyzed using Bonferroni-adjusted $\alpha$ levels

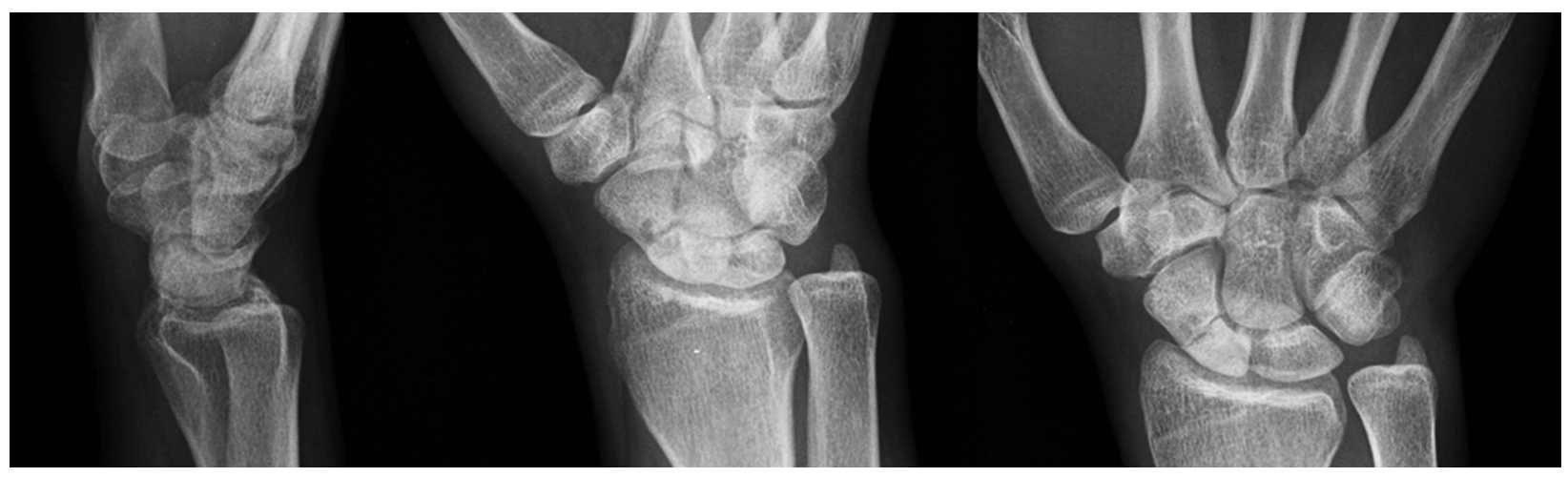

Fig. 1 Preoperative computed tomography scan and X-ray images of a delayed union of a left scaphoid. 


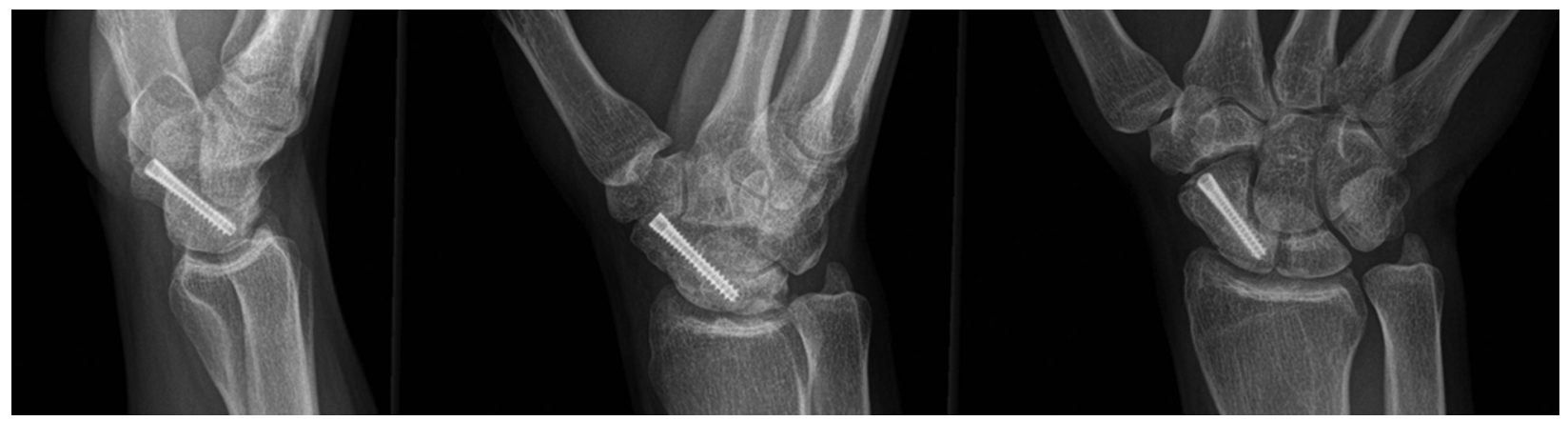

Fig. 2 X-ray image after percutaneous fixation of the scaphoid showing union of the fracture site.

of 0.007 per parameter $(0.05 / 7)$ to correct for multiple comparisons.

\section{Results}

Sixteen patients with late presentation of a scaphoid fracture were treated with percutaneous screw fixation without bone graft (Appendix). All included patients attended the final review appointment. Union was obtained in 15 (94\%) of the 16 patients at a mean of 4 months following surgery (range: 1-9 months). There was no correlation between the time to surgery and the time to union $(R=-0.298 ; p=0.281)$. In one patient, the scaphoid classified as a grade 3 nonunion 27 weeks after initial injury failed to unite. The revision procedure in this patient was performed 19 months after the initial surgery, and union of the scaphoid was achieved in 6 months with CT scan assessment.

Average follow-up time was 36 months (range: 12-98 months). The average movement arcs and grip strength are summarized in - Table 1. No significant statistical difference was found in ROM or grip strength between the operated and contralateral wrist.

The average postoperative DASH score was 6 (range: 0-39). The average postoperative PRWHE score was 10 (range: 0-56). According to the DASH questionnaire, 12 patients did not report any wrist pain at rest. The patient with failure of scaphoid union complained of moderate wrist pain, and one other patient reported mild wrist pain. No complications from the percutaneous technique were encountered. Scaphotrapezial arthritis progression was never noted in this series.

\section{Discussion}

Volar percutaneous screw fixation without bone graft was commonly used for acute scaphoid fractures with good reported outcomes. ${ }^{1,16-18}$ However, limited data of the volar percutaneous screw fixation were described in the late presentations of the scaphoid fractures. Kim et $\mathrm{al}^{8}$ included 12 patients and found a $100 \%$ union rate at 12 months followup with an average time to union after surgery of 3 months and the average DASH score of 9 (range: 0-21). Capo et $\mathrm{al}^{7}$ used a volar percutaneous approach for scaphoid nonunion in 8 of 12 patients and obtained a union rate of $92 \%$ with an average time to union of 3 months and the average DASH score of 6 (range: 0-16) at final follow-up.

In this study, a union rate of $94 \%$ was achieved using a volar percutaneous transtrapezial screw fixation technique without bone graft. ${ }^{14}$ There was no significant difference in ROM or grip strength between the operated side and the contralateral side at the final follow-up. The average time to union was 4 months and the average DASH score was 6 (range: 0-39). The technique allows placement of the screw exactly along the central axis of the scaphoid, which may correlate to biomechanical superiority. ${ }^{19}$ This technique may not require dividing the volar carpal ligaments nor damage the blood supply of the scaphoid, and may result in a smaller scar.

Drilling of the scaphotrapezial joint did not lead to symptomatic osteoarthritis in this study, which was consistent with a short- to medium-term follow-up by Geurts et al. ${ }^{17}$

Table 1 Average movement arcs (degrees) and grip strength (kg)

\begin{tabular}{|l|l|l|l|}
\hline & Injured & Uninjured & $p$-Values \\
\hline Average flexion arc & 83 degrees (range: $68-110)$ & 87 degrees (range: $68-110)$ & 0.138 \\
\hline Average extension arc & 71 degrees (range: $36-100)$ & 77 degrees (range: $52-110)$ & 0.083 \\
\hline Average pronation arc & 85 degrees (range: $75-92)$ & 87 degrees (range: $80-100)$ & 0.114 \\
\hline Average supination arc & 89 degrees (range: $80-100)$ & 90 degrees (range: $86-92)$ & 0.577 \\
\hline Average radial deviation arc & 27 degrees (range: $10-42)$ & 28 degrees (range: $19-38)$ & 0.798 \\
\hline Average ulnar deviation arc & 54 degrees (range: $36-70)$ & 54 degrees (range: $38-70)$ & 0.838 \\
\hline Average grip strength & 43 degrees (range: $23-58)$ & 45 degrees (range: $25-59)$ & 0.244 \\
\hline
\end{tabular}

Note: This table includes the average movements arcs and grip strength of the injured and uninjured wrist. 
Careful evaluation of late presentations of scaphoid fractures, and patient selection for the procedure are important. CT scan has been shown to be superior to radiographs for the evaluation of scaphoid fracture displacement and union. ${ }^{20,21}$ Therefore, this is often indicated when radiographs are not clear enough for final decision making. In this study, CT scans of the scaphoid were deemed necessary in eight patients to evaluate cyst formation, sclerosis, and fracture displacement and to decide on further treatment. Patients with delayed union of the scaphoid with or without cyst formation are good candidates for percutaneous screw fixation, as long as there are no signs of sclerosis or cortical bone resorption with fracture displacement.

Slade and Geissler proposed a revised classification of scaphoid nonunion in an effort to match the healing potential of a nonunion to a specific treatment algorithm. ${ }^{13}$ Nonunion of grade I (fibrous union), grade II (fibrous nonunion with minimal sclerosis, $<1 \mathrm{~mm}$ ), and grade III (minimal bone resorption of the anterior cortical bone and limited fracture sclerosis, $<2 \mathrm{~mm}$ ) were found appropriate for rigid fixation without bone grafting. In this study, excellent clinical outcome was obtained with the technique for grades I to III.

This study has several limitations. First, our sample size was small because late presentations of scaphoid fractures were not encountered very often. Second, the time to union was assessed clinically and radiographically in 4 week intervals. Third, CT scans were not performed to confirm union of all scaphoids. In the postoperative follow-up, we will try to avoid unnecessary exposure to radiation. We will therefore only ask for postoperative CT scans when radiographs do not show progressive healing, when signs of loosening around the screw are present, or when patient symptoms do not resolve. Fourth, it is a retrospective study and no control group was available. For clinical evaluation, the contralateral hand was used to assess functional outcome.

Percutaneous screw fixation of the scaphoid was successful in carefully selected cases of scaphoid delayed presentation, delayed union, or nonunion. Cyst formation, less than $5 \mathrm{~mm}$, is not a contraindication as long as there are no signs of fracture displacement. We do not recommend this technique if there is sclerosis or collapse at the scaphoid delayed presentation, delayed union, or nonunion site.

\section{Conflict of Interest}

None.

\section{Acknowledgment}

The authors thank Kristien Vuylsteke (MoRe Foundation) for her help with the statistical analysis.

\section{References}

1 Bond CD, Shin AY, McBride MT, Dao KD. Percutaneous screw fixation or cast immobilization for nondisplaced scaphoid fractures. J Bone Joint Surg Am 2001;83-A(4):483-488
2 Chechik O, Rosenblatt Y. Management of clinically suspected scaphoid fractures: a survey of current practice in Israel. Isr Med Assoc J 2009;11(4):225-228

3 Osterman AL, Mikulics M. Scaphoid nonunion. Hand Clin 1988; 4(3):437-455

4 Merrell G, Slade J. Technique for percutaneous fixation of displaced and nondisplaced acute scaphoid fractures and select nonunions. J Hand Surg Am 2008;33(6):966-973

5 Grewal R, Suh N, MacDermid JC. The missed scaphoid fractureoutcomes of delayed cast treatment. J Wrist Surg 2015;4(4): 278-283

6 Ibrahim T, Qureshi A, Sutton AJ, Dias JJ. Surgical versus nonsurgical treatment of acute minimally displaced and undisplaced scaphoid waist fractures: pairwise and network meta-analyses of randomized controlled trials. J Hand Surg Am 2011;36(11):1759-1768.e1

7 Capo JT, Shamian B, Rizzo M. Percutaneous screw fixation without bone grafting of scaphoid non-union. Isr Med Assoc J 2012;14(12): 729-732

$8 \mathrm{Kim}$ JK, Kim JO, Lee SY. Volar percutaneous screw fixation for scaphoid waist delayed union. Clin Orthop Relat Res 2010;468(4): 1066-1071

9 Inaparthy PK, Nicholl JE. Treatment of delayed/nonunion of scaphoid waist with Synthes cannulated scaphoid screw and bone graft. Hand (NY) 2008;3(4):292-296

10 Slade JF III, Dodds SD. Minimally invasive management of scaphoid nonunions. Clin Orthop Relat Res 2006;445(445):108-119

11 Vinnars B, Pietreanu M, Bodestedt A, Ekenstam Fa, Gerdin B. Nonoperative compared with operative treatment of acute scaphoid fractures. A randomized clinical trial. J Bone Joint Surg Am 2008;90(6):1176-1185

12 Herbert TJ, Fisher WE. Management of the fractured scaphoid using a new bone screw. J Bone Joint Surg Br 1984;66(1):114-123

13 Slade JF III, Geissler WB, Gutow AP, Merrell GA. Percutaneous internal fixation of selected scaphoid nonunions with an arthroscopically assisted dorsal approach. J Bone Joint Surg Am 2003;85A(4, Suppl 4):20-32

14 Geurts GF, Van Riet RP, Meermans G, Verstreken F. Volar percutaneous transtrapezial fixation of scaphoid waist fractures: surgical technique. Acta Orthop Belg 2012;78(1):121-125

15 Brown GD III, Roh MS, Strauch RJ, Rosenwasser MP, Ateshian GA, Mow VC. Radiography and visual pathology of the osteoarthritic scaphotrapezio-trapezoidal joint, and its relationship to trapeziometacarpal osteoarthritis. J Hand Surg Am 2003;28(5):739-743

16 Adolfsson L, Lindau T, Arner M. Acutrak screw fixation versus cast immobilisation for undisplaced scaphoid waist fractures. J Hand Surg [Br] 2001;26(3):192-195

17 Geurts G, van Riet R, Meermans G, Verstreken F. Incidence of scaphotrapezial arthritis following volar percutaneous fixation of nondisplaced scaphoid waist fractures using a transtrapezial approach. J Hand Surg Am 2011;36(11):1753-1758

18 McQueen MM, Gelbke MK, Wakefield A, Will EM, Gaebler C. Percutaneous screw fixation versus conservative treatment for fractures of the waist of the scaphoid: a prospective randomised study. J Bone Joint Surg Br 2008;90(1):66-71

19 Meermans G, Van Glabbeek F, Braem MJ, van Riet RP, Hubens G, Verstreken F. Comparison of two percutaneous volar approaches for screw fixation of scaphoid waist fractures: radiographic and biomechanical study of an osteotomy-simulated model. J Bone Joint Surg Am 2014;96(16):1369-1376

20 Buijze GA, Jørgsholm P, Thomsen NO, Bjorkman A, Besjakov J, Ring D. Diagnostic performance of radiographs and computed tomography for displacement and instability of acute scaphoid waist fractures. J Bone Joint Surg Am 2012;94(21):1967-1974

21 Lozano-Calderón S, Blazar P, Zurakowski D, Lee SG, Ring D. Diagnosis of scaphoid fracture displacement with radiography and computed tomography. J Bone Joint Surg Am 2006;88(12): 2695-2703 


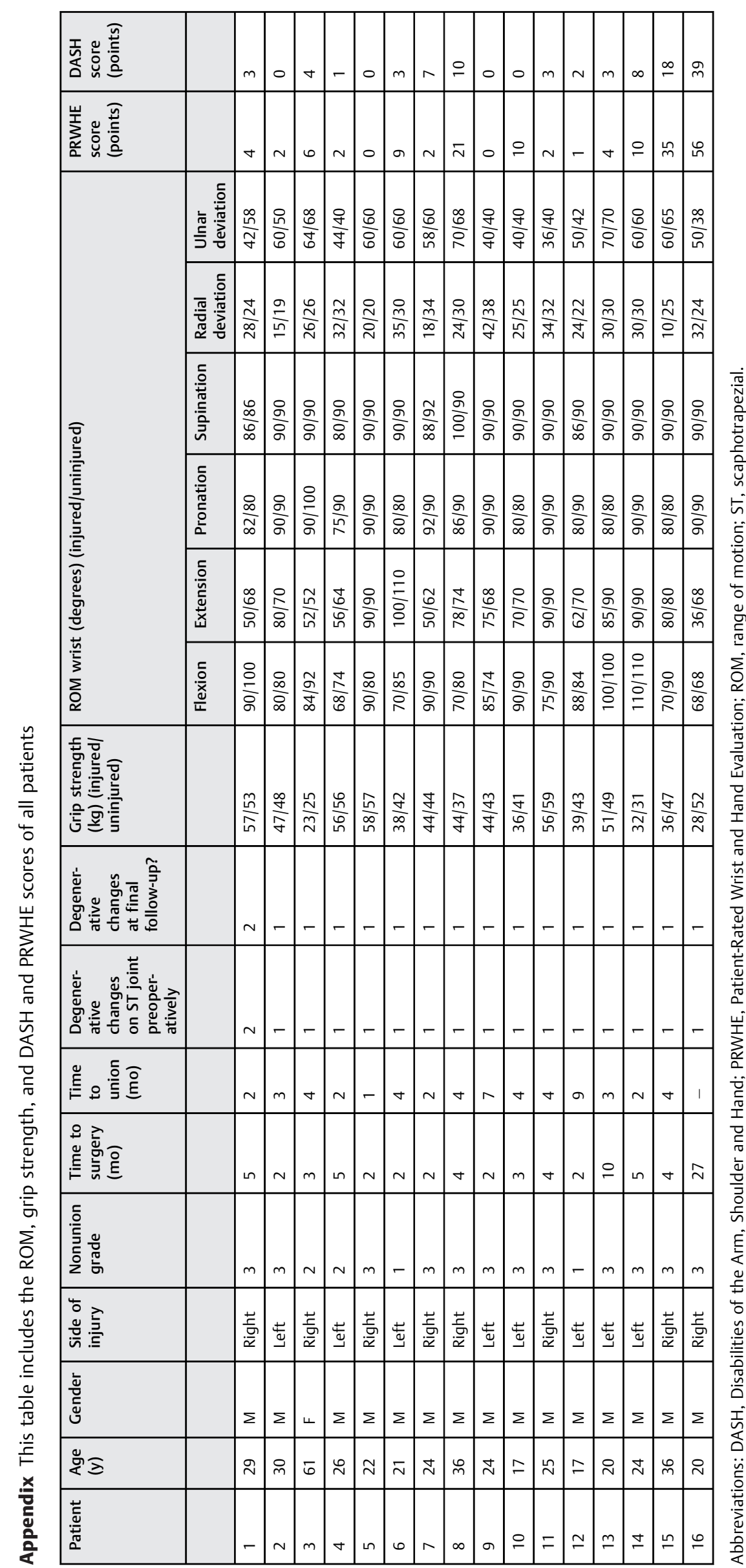

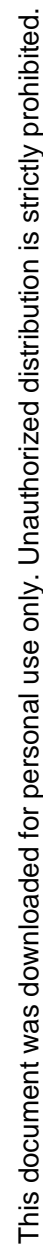

